\title{
Cálculos químicos quânticos e seus usos
}

\section{Quantum chemical calculations and their uses}

Cálculos químicos cuánticos y sus usos

Paulo de Tarso Ferreira Sales

ORCID: https://orcid.org/0000-0002-0030-0316

Universidade Federal de Goiás, Brasil

E-mail: paulogyn8@gmail.com

Katia Maria de Souza

ORCID: https://orcid.org/0000-0001-7311-1762

Centro Universitário - UniBrasília, Brasil

E-mail: engkatia@gmail.com

Alyne Gonçalves Bezerra

ORCID: https://orcid.org/0000-0001-9270-7844

Universidade Federal de Goiás, Brasil

E-mail: alyneufg@gmail.com

Satu Anneli Ojala

ORCID: https://orcid.org/0000-0001-9520-9293

Universidade de Oulu, Finlândia

E-mail: satu.ojala@oulu.fi

Sérgio Botelho de Oliveira

ORCID: https://orcid.org/0000-0003-0961-938X

Instituto Federal de Goiás, Brasil

E-mail: sergio.oliveira@ifg.edu.br

Maria Teresa Freitas Bara

ORCID: https://orcid.org/0000-0003-4942-8721

Universidade Federal de Goiás, Brasil E-mail: mtbara@gmail.com

\section{Resumo}

Neste trabalho, apresentamos estudos que utilizaram a química quântica como uma ferramenta de previsão comportamentos da química e da física molecular, que forneceram dados altamente precisos e quantitativos sobre sistemas moleculares. Iniciamos por rever a teoria da estrutura eletrônica baseada na função das ondas, enfatizando a hierarquia N-eletrônica da teoria dos pares-cluster e a hierarquia de um electrão de conjuntos de bases consistentes de correlação. Posteriormente, mostramos estudos realizados em diversas áreas da química em que os dados quânticos foram usados para estudar comportamentos de compostos em diversas áreas da química moderna. Uma grande vantagem do uso da química quântica computacional é a possibilidade de estudar mecanísticas que não são experimentalmente viáveis de serem executadas, mas os dados fornecidos por ela garantem grande confiabilidade dos dados apresentados. A química computacional se tornou uma ferramenta de grande utilidade na química moderna.

Palavras-chave: Teoria do funcional da densidade; Espectrometria de massas; Espectroscopia de infravermelho e Raman; Carbonização hidrotermal; Catálise heterogênea.

\begin{abstract}
In this work, we present studies that used quantum-chemical calculation as a tool for predicting behaviors in chemistry and molecular physics, which provided highly accurate and quantitative data on molecular systems. We start by reviewing the theory of electronic structure based on the wave function, emphasizing the $\mathrm{N}$-electronic hierarchy of the pair-cluster theory and the one-electron hierarchy of sets of consistent bases of correlation. Later, we show studies carried out in different areas of chemistry in which quantum data were used to study the behavior of compounds in different areas of modern chemistry. A great advantage of the use of computational quantum chemistry is the possibility of studying mechanistics that are not experimentally feasible to be performed, but the data provided by it guarantee great reliability of the presented data. Computational chemistry has become a very useful tool in modern chemistry.
\end{abstract}

Keywords: Density functional theory; Mass spectrometry; Infrared and Raman spectroscopy; Hydrothermal carbonization; Heterogeneous catalysis.

\section{Resumen}

En este trabajo, presentamos estudios que utilizaron la química cuántica como herramienta para predecir comportamientos en química y física molecular, lo que proporcionó datos cuantitativos y de alta precisión sobre 
sistemas moleculares. Comenzamos revisando la teoría de la estructura electrónica basada en la función de onda, enfatizando la jerarquía $\mathrm{N}$-electrónica de la teoría de pares-cúmulos y la jerarquía de un electrón de conjuntos de bases consistentes de correlación. A continuación, mostramos estudios realizados en diferentes áreas de la química en los que se utilizaron datos cuánticos para estudiar el comportamiento de compuestos en diferentes áreas de la química moderna. Una gran ventaja del uso de la química cuántica computacional es la posibilidad de estudiar mecánicas que no son factibles de realizar experimentalmente, pero los datos que proporciona garantizan una gran fiabilidad de los datos presentados. La química computacional se ha convertido en una herramienta muy útil en la química moderna.

Palabras clave: Teoría funcional de la densidad; Espectrometría de masas; Espectroscopía infrarroja y Raman; Carbonización hidrotermal; Catálisis heterogénea.

\section{Introdução}

O desenvolvimento química quântica mostrou-se uma ferramenta versátil, cuja aplicação em muitas áreas da química e a física fornece-nos dados quantitativos sobre química, ajudando-nos a prever, confirmar, ou rejeitar observações e medições experimentais (Helgaker et al., 2008). Contudo, essa ferramenta ainda é pouco usada na predição e estudos na área da química molecular e com isso, é nosso propósito aqui para rever a química quântica enquanto tal um instrumento quantitativo, capaz de produzir numéricos resultados que concordam quantitativamente com o verdadeiro ou exato resultados, para dentro de alguma incerteza estimada. Bem como mostrar os resultados já publicados em que essa ferramenta foi usada.

Iniciaremos mostrando os fundamentos da química quântica mostrando que na década de 1930, James e Coolidge utilizados uma forma funcional semelhante, incluindo todos os poderes do $\mathrm{r}_{12}$ para cálculos sobre o estado de base das moléculas hidrogênio, alcançando precisão de millihartree (Coolidge \& James, 1938). Após esse estudo, particularmente importantes foram o desenvolvimento da teoria do grupamento de clusters desde o no início dos anos 80 por Bartlett (1995) e o desenvolvimento dos conjuntos de bases de correlação consistentes desde o final dos anos 80 por Ferrari e Bennett (2019).

A abordagem quântico química padrão de alta precisão baseia-se no conceito de teoria em que o estado eletrônico é representado por um conjunto de orbitais moleculares ocupadas (Helgaker et al., 2008). Com isso, a teoria do estado fundamental descrita pela função de onda Hartree-Fock (obtida minimizando o valor da expectativa do eletrônico de energia de uma função de onda determinante), que é a melhor aproximação do estado eletrônico do estado fundamenta e foi descrita como:

$$
\langle H F|\hat{H}| H F\rangle=\langle\min \Psi \operatorname{det}|\hat{H}| \Psi \text { det }\rangle
$$

Com o passar dos anos, várias modificações e formulações alternativas da teoria do agrupamento acoplado foram propostas, sendo que uma dessas modificações populares da teoria do acoplamento padrão de agrupamento cluster (Pople et al., 1987), da expansão da onda parcial (Helgaker et al., 2014) (em que a função de onda de hélio no estado fundamental pode ser escrita como um produto de uma parte espacial simétrica e um parte de centrifugação antissimétrica), da expansão primordial (Kutzelnigg \& Morgan III, 1992), em que num finito conjunto de funções de um único elétron ele insere um conjunto adicional de erro na descrição, para além da expansão de onda parcial na descrição espacial radial proposta por Löwdin (1955), dos conjuntos de base (basis set) molecular gaussianos (em que todas as funções de ondas moleculares são usadas nos cálculos) (Helgaker et al., 2014), dos orbitais atômicos naturais em que Almlöf e Taylor (1987) propuseram gerar conjuntos de base para cálculos correlacionados a partir de orbitais atômicos naturais, obtidos por diagonalização a matriz de densidade de um elétron a partir de uma matriz atômica (Almlöf \& Taylor, 1991); do conjuntos de base consistentes com correlação, em que é sugerido que pode ser possível construir conjuntos de base para a correlação dos elétrons a partir do perfil energético (Almlöf \& Taylor, 1991), sendo que cada orbital correlativo é representado por um único gaussiano primitivo escolhido para maximizar a sua contribuição para a energia de correlação e onde todos os orbitais que fazem contribuições semelhantes (Dunning Jr, 1989); da extrapolação dos conjuntos de base, em que em um sistema molecular, a convergência das bases pode ser afetada por outros aspectos da estrutura electrónica (tal como a polarização do distribuições de cargas atómicas 
na presença de outros átomos e campo eletromagnético externo) (Bakowies, 2007a, 2007b).

Desde o início dos estudos sobre quântica, o fato da correlação dinâmica é conhecida como um problema a ser resolvido. Contudo, somente com James e Coolidge (1936) coordenadas elípticas foram utilizadas e as integrais de Coulomb foram avaliados por meio da expansão de Neumann. A fim de resolver a integral de quatro elétrons, Szalewicz et al. (1982) propôs o funcional da fraca ortogonalidade, sendo que a avaliação direta de os numerosos integrais de três e quatro elétrons foram evitadas, reduzindo avaliação integral a, no máximo, dois integrais de elétrons. A ideia de métodos transcorrelacionados foi empregado por Imamura e Scuseria (2003) para desenvolver uma nova correlação funcional para a teoria do funcional da densidade (TFD). Correções não harmônicas foram feitas usando a TFD, para calcular a energia de atomização de um pequeno hidrocarboneto, que foi na ordem de 1-2 kJ (Daniel Boese et al., 2005).

$\mathrm{O}$ conceito TFD baseia-se no primeiro teorema de Hohenberg-Kohn, que afirma que a densidade de elétrons $\mathrm{F}_{(\mathrm{r})}$ determina o potencial externo (isto é, devido ao núcleo) por normalização (Geerlings et al., 2003):

$$
\int \rho_{(\mathrm{r})} \mathrm{d}_{\mathrm{r}}=\mathrm{N} \text { (Equação 2) }
$$

$\rho(\mathrm{r})$ é a densidade eletrônica,

$\mathrm{N}$ é o número de elétrons,

r é o potencial externo exercido pelo núcleo.

A partir do uso da TFD, avanços significativos foram feitos no sentido de aprimorar os cálculos dos integrais, mas eles ainda são relativamente demorados (Fonseca Guerra et al., 1998). Nos últimos dez anos, majoritariamente as publicações, de acordo com o site scholar Google, foram desenvolvidos pelo nível de teoria TFD (Figura 1).

Figura 1. Número de publicações em que TFD e PM2 foram citadas, de acordo com a busca do scholar google.

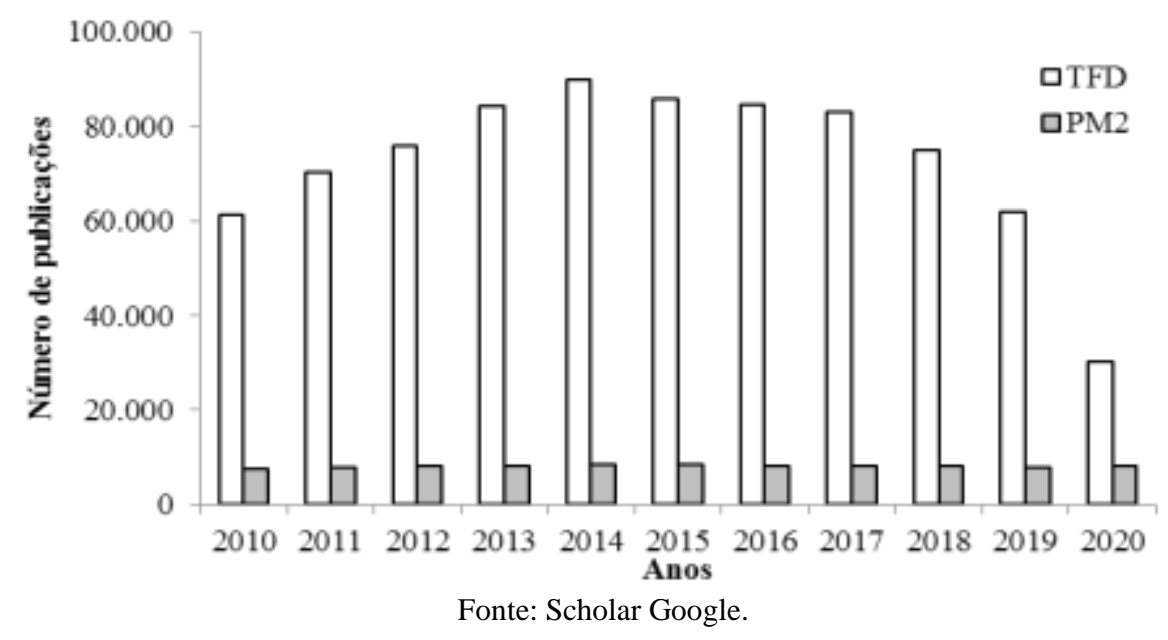

\section{Metodologia}

O seguinte estudo tem caráter descritivo com abordagem qualitativa de artigos publicados sobre a química quântica computacional e a busca foi efetuada utilizando-se o aplicativo do Google chamado Scholar Google. O software Gaussian 16 revisão A.03 (Frisch et al., 2016) foi usado para realizar os cálculos de química quântica. As estruturas moleculares de todas as espécies estado fundamental foram otimizadas usando o nível B3LYP, sendo que as geometrias foram otimizadas para obter energia mínima local. Imagens de estruturas moleculares e o espectro infravermelho foram geradas usando o software Avogadro, versão 1.20 (Hanwell et al., 2012). A metodologia de pesquisa utilizada foi sugerida por Pereira et al. (2018) e 
Köche (2016).

\section{Resultados e Discussão}

\subsection{Estudos sobre Espectrometria de Massas e Quântica}

A TFD da tem sido utilizado para estudar o processo de ionização molecular e subsequentes quebras de ligação em estudos de espectrometria de massas - EM. De acordo com Alex et al. (2009), a previsão da fragmentação da EM utilizando o método TFD indicou que ela era a ferramenta mais inteligente e fiável para apresentar dados correlatos aos dados experimentais. Além disso, a migração do hidrogênio para o-carboneto próximo da ligação amida é considerada o principal mecanismo de fragmentação da EM (Kenny et al., 1992). Maclot et al. (2013) mostraram que a migração intramolecular ultrarrápida de hidrogênio compete com a esperada repulsão colúmbica, mas a presença de uma frequência imaginária específica representa um excelente método para identificar os estados de transição, que corresponde ao modo de resposta (Song et al., 2018). Estudando a migração intramolecular no processo de fragmentação em EM, Alsheikh et al. (2015) relataram que átomos de hidrogênio com cargas atômicas próximas de zero eram capazes de migrar e que a fraca interação eletrostática entre átomos de hidrogênio e carbono favorecem a migração. (Vessecchi et al., 2017) usaram a teoria quântica para entender o enfraquecimento e o fortalecimento das ligações atômicas e a influência das mesmas no processo de fragmentação em EM.

O Quadro 1 lista vários trabalhos que envolveram a espectrometria de massas e TFD, na tentativa de elucidação da estrutura molecular.

Quadro 1 - Estudos publicados sobre EM e cálculos quânticos.

\begin{tabular}{|l|l|}
\hline Assunto & Autores \\
\hline Identificação de polifenóis em flores de Phoenix dactylifera. & Ben Said et al. (2017) \\
\hline Diferenciação entre D e L isoleucina. & S.-S. Lee et al. (2018) \\
\hline Rotas de reação baseadas na teoria gráfica para os estudos de mecanismo de sequenciação de & J. Lee et al. (2020) \\
\hline Análise de anômeros e confôrmero de monossacarídeos. & Barnes et al. (2020) \\
\hline Estudo da protonação e decomposição de tirosina. & Bahrami et al. (2018) \\
\hline Caracterização de (4-amino-2-(fenilamina)tiazol-5-il)(tiofeno-2-il)metanona e (4-amino-2-(4- & Shahana e Yardily, (2020) \\
\hline Estudo da Complexação de uranila por N-2-hidroetil. & Sharma et al. (2020) \\
\hline Síntese e caracterização espectral do corante aminometilbenzóico. & Prashantha et al. (2021) \\
\hline Elucidação do espectro de massas do alcalóide retronecina. & Modesto-Costa et al. (2018) \\
\hline
\end{tabular}

Fonte: Scholar Google.

Como pode ser observado, os cálculos quânticos podem ser usados no processo de entendimento da fragmentação, do comportamento químico e da estrutura molecular de compostos que foram submetidos à EM. A Figura 2 mostra a estrutura molecular do aduto de glimeperida sodiadada. 
Figura 2 - Estrutura geométrica molecular otimizada de glimeperida calculado no nível B3LYP/cc-pvdz. Os átomos são numerados e rotulados, sendo que os cálculos quânticos foram feitos no programa Gaussian 16 e a imagem foi gerada pelo programa Avogadro.

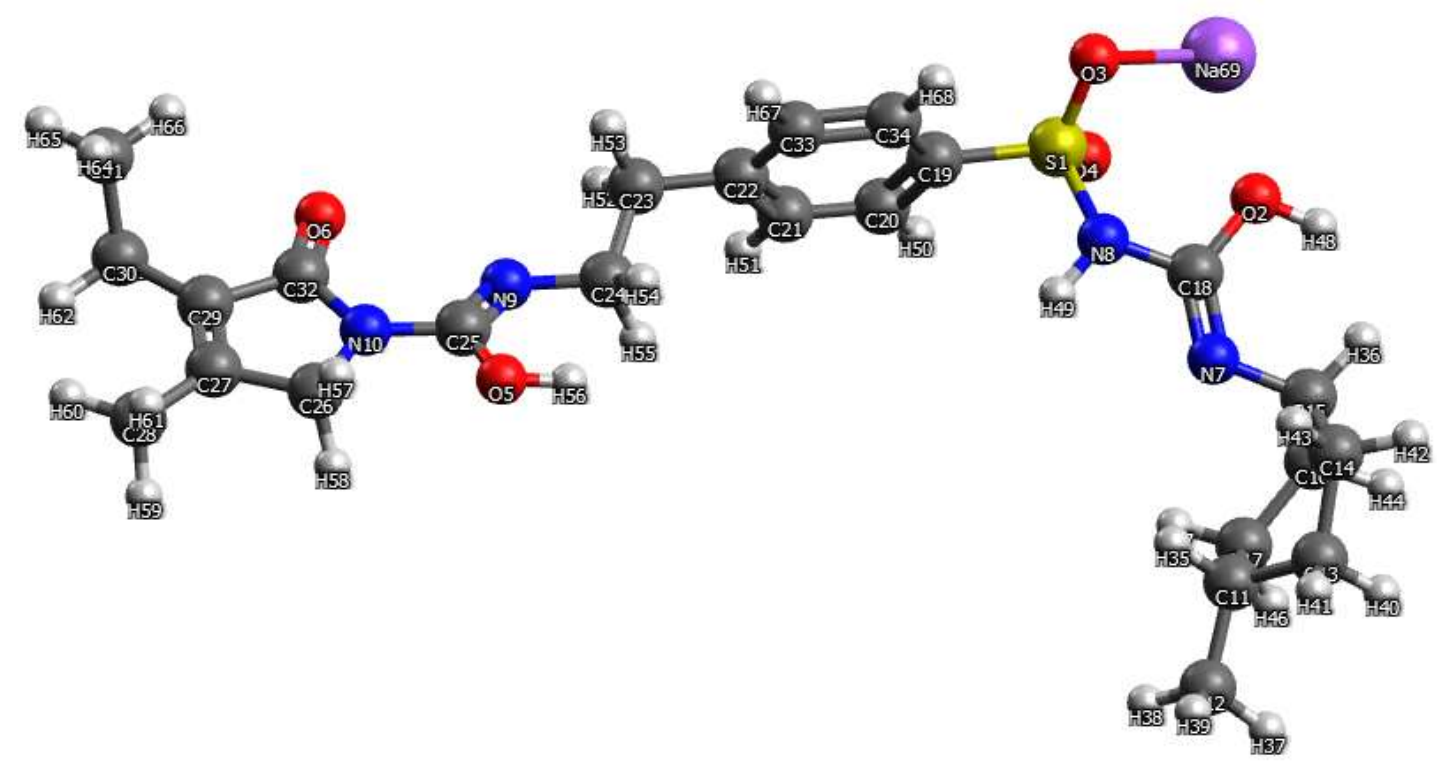

Fonte: Autores.

\subsection{Radiação Infravermelha e Espectroscopia Raman e Cálculos Quânticos}

O uso de métodos analíticos modernos que utilizam a espectroscopia infravermelha apresentam grandes vantagens, como a facilidade de instrumentação, baixo tempo analítico e baixo custo fazem com que esses métodos sejam (Huck, 2017). Além disso, é bem sabido que a DFT fornece importantes informações sobre a estrutura molecular e suas interações (Rad et al., 2021). A partir das interações, o uso da radiação infravermelha e a TFD se complementaram a fim de estudar as estruturas moleculares de compostos.

As equações de Newton para a oscilação molecular, na qual as frequências vibracionais harmônicas são obtidas através da diagonalização da matriz de segundas derivadas da massa com a energia potencial (Beć \& Huck, 2019). Contudo, esses cálculos são aplicáveis somente a sistemas mais simples (Polyansky et al., 2003). O Quadro 2 mostra os estudos que usaram infravermelho e TFD em seus estudos.

Quadro 2 - Estudos espectros sobre espectroscopia de infravermelho e Raman e cálculos quânticos.

\begin{tabular}{|l|l|}
\hline Assunto & Autores \\
\hline Espectroscopia de infravermelho de Favipiravir & Rad et al., 2021 \\
\hline Equilíbrio conformacional de álcool butílico & Grabska et al., 2017 \\
\hline Propriedades ópticas de corante ciano/óxido de zinco & Al-Hossainy et al., 2021 \\
\hline Síntese, caracterização e avaliação da oxidação de azul de bromotimol & Ibrahim \& Al-Hossainy, 2021 \\
\hline Correlação espectro/estrutura de n-hexano, ciclohexanol e fenol & Beć et al., 2018 \\
\hline Saturação e insaturação de ácidos graxos de cadeia longa & Grabska et al., 2018 \\
\hline Síntese e estudos espectroscópicos de um híbrido indol-isatino & Almutairi et al., 2018 \\
\hline Análise espectroscópica e propriedades de derivados de imidazol & Thomas et al., 2018 \\
\hline $\begin{array}{l}\text { Síntese e estudo molecular e atividade antibacteriana de um ligante de hidrazona à base de } \\
\text { xanteno }\end{array}$ & Naseem et al., 2017 \\
\hline $\begin{array}{l}\text { O espectro de infravermelho independente de cada componente em N-metilacetamida foi } \\
\text { calculado usando o método da TFD e a contribuição de cada componente para as bandas de } \\
\text { amida I, II e III foi analisada. }\end{array}$ & Ji et al \\
\hline
\end{tabular}


Diferentemente, a espectroscopia comumente aplicada baseia-se em métodos não harmónicos razoavelmente eficientes com a capacidade de tratar moléculas complexas (Beć \& Huck, 2019). Com isso, uma determinação altamente precisa dos estados vibracionais e das intensidades de transição, por exemplo, em exames sistemáticos de variações espectrais subtis devido a certos substitutos foi reportada (Takahashi \& Yabushita, 2013). Além disso, uma modelagem molecular permitiu estudar sobre a influência da constante dielétrica do solvente no modo de estiramento X-H na fase de solução, como relatado por (Futami et al., 2011).

Como pode ser visto, o uso da TFD tem grande uso para desenvolvimentos de métodos analíticos a partir da radiação infravermelha. Como exemplo da versatilidade dos cálculos quânticos para métodos vibracionais, a Figura 3 mostra o espectro infravermelho teórico do aduto de sódio de glimeperida.

Figura 3 - Espectro teórico infravermelho calculado no nível B3LYP/cc-pvdz, calculado pelo programa Gaussian 16 e a imagem foi gerada pelo programa Avogadro.

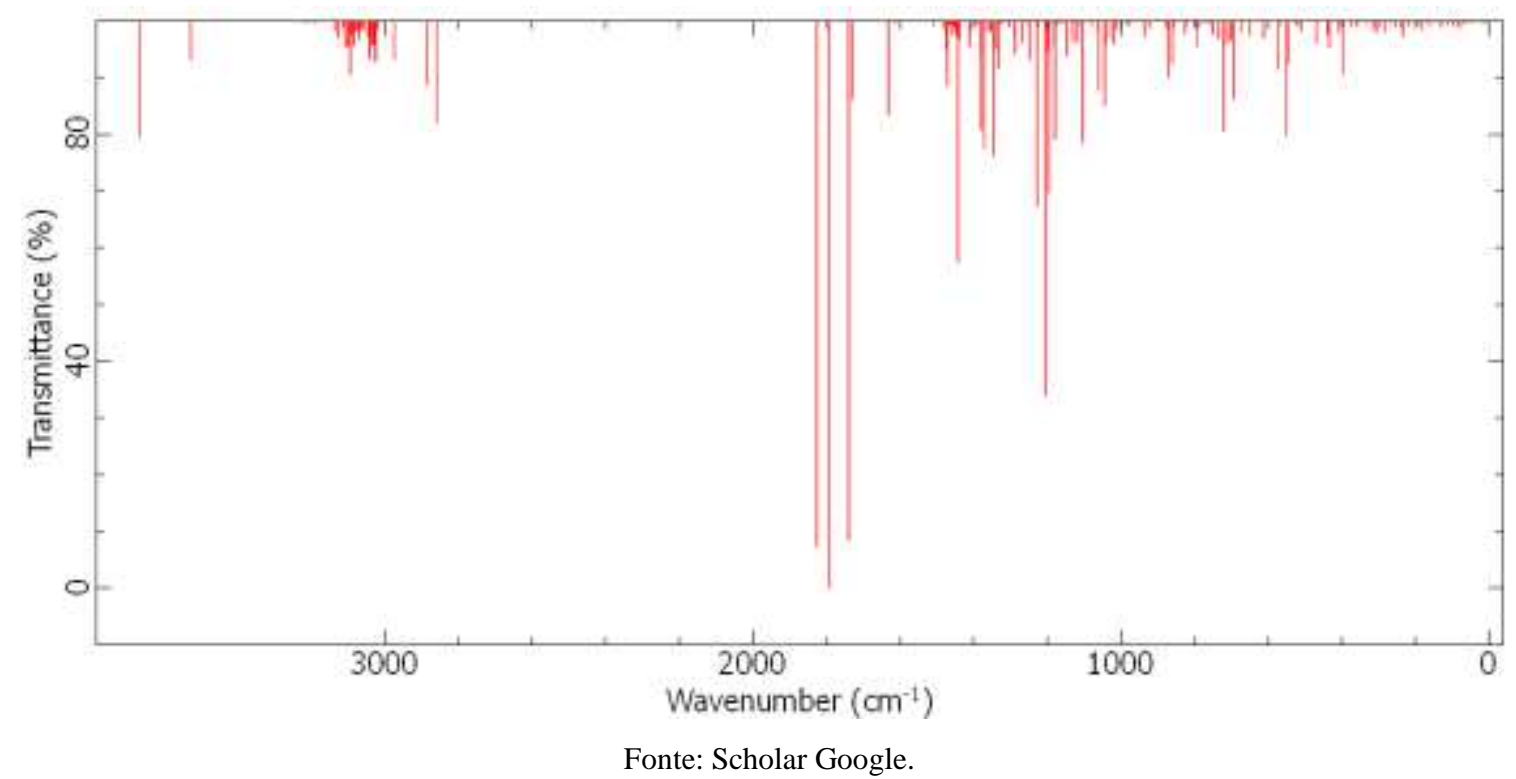

\subsection{Carbonização Hidrotermal}

Um método ambientalmente amigável de produção de produtos carbonáceos a partir de fontes sustentáveis é a carbonização hidrotermal (CHT). Ele é um processo térmico em que é possível converter biomassa em produtos com valor commercial (Xiao et al., 2012). Esse método é muito interessante, pois o compost final, chamado de hydrochar, é capaz de captutur o carbono (Titirici et al., 2007). Mais que isso, a alta versatilidade de converter lodo de estações de tratamento de esgoto (He et al., 2013), biomassa lignocelulósica (Antero et al., 2019; Mäkelä et al., 2015), lodo de estação de tratamento de indústria papeleira (Lin et al., 2015) e microalgas (Heilmann et al., 2011). Devido à grande área superficial e porosidade, hydrochars tem sido descritos como materiais para aramazenamento de energia (Jain et al., 2016), energia eletroquímica (Fan et al., 2015) e gases (Morris \& Wheatley, 2008). Além disso, novos materiais foram sintetizados a partir de hydrochar, como: carbvão ativado (Başakçılardan Kabakcı \& Baran, 2019; Sanjeev Sharma \& Chun, 2019) e supercapacitores (Ding et al., 2012, 2013; Gao et al., 2015). A Figura 4 mostra o esquema de produção de hydrochar. 
Figura 4 - Esquema de produção de hydrochar.

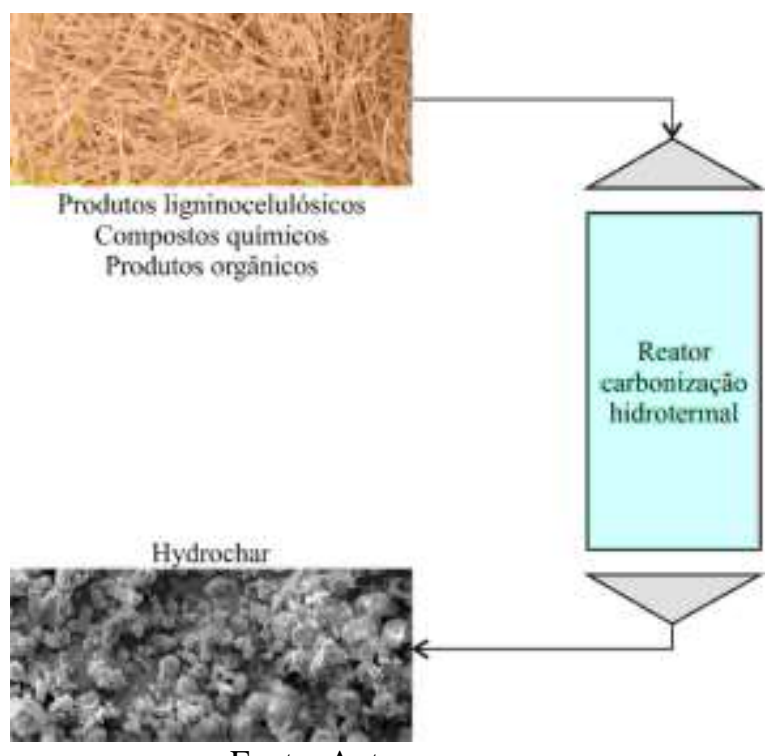

Fonte: Autores.

Cálculos quânticos foram usados para entender vários aspectos da CHT e da formação do hydrochar, como é mostrado no Quadro 3.

Quadro 3 - Estudos sobre CHT e cálculos quânticos.

\begin{tabular}{|l|l|}
\hline Assunto & Autores \\
\hline $\begin{array}{l}\text { A estrutura molecular otimizada, a energia potencial e o momento de dipolo de pontos quânticos } \\
\text { de carbono (gerados por CHT) foram estudados usando TFD. }\end{array}$ & Jamaludin et al., 2020 \\
\hline $\begin{array}{l}\text { Otimização da geometria e cálculo de orbital integrado pi localizado sobre o plano de material } \\
\text { derivado de cigarro, submetido à CHT. }\end{array}$ & Shao et al., 2021 \\
\hline Modelagem molecular de hydrochar e teórico espectro Raman e infravermelho. & Brown et al., 2017 \\
\hline $\begin{array}{l}\text { Os cálculos da TFD mostraram que um biochar gerado por CHT a partir de retardantes de } \\
\text { chamas organofosfatados aromáticos pode atrair espécies protonadas. }\end{array}$ & Du et al., 2020 \\
\hline $\begin{array}{l}\text { A distribuição do tamanho de poro do hydrochar foi estimada a partir do modelo TFD, sendo que } \\
\text { o material foi sintetizado a partir de sobras de mandioca. }\end{array}$ & Wu et al., 2020 \\
\hline $\begin{array}{l}\text { A adsorção de iodeto de hidrogênio nas superfícies de carbono N-livres e N-dopados foram } \\
\text { estudadas por TFD e hydrochar foi sintetizado a partir de cascas de coco. }\end{array}$ & X. Li et al., 2020 \\
\hline $\begin{array}{l}\text { A distribuição do tamanho e o volume dos poros foram derivados da adsorção da isotérmica } \\
\text { usando a TFD, de um hydrochar gerado a partir de folhas de cânfora }\end{array}$ & Guangzhi et al., 2017 \\
\hline
\end{tabular}

Fonte: Scholar Google.

\subsection{Superfícies Catalíticas}

Estudar as taxas de reações catalíticas modeladas computacionalmente para reproduzir o arranjo de átomos da superfícies a partir de cristais é um dos grandes triunfos da ciência moderna (Jinnouchi \& Asahi, 2017). Quando se fala em reações catalíticas, reações de transferência de cargas de óxidos de nitrogênio (Sibari et al., 2021), de adsorção de CO (Yang et al., 2019), de adsorção de água (Shi et al., 2020) e hidrogênio (Gómez et al., 2017), de adsorção de sais de amida e amônio (Chen et al., 2017), esses estudos demonstram que a catálise heterogênea pode ser amplamente estudada usando a TFD. Atualmente, o programa usado para os cálculos quânticos é o Gaussian 16 software (Frisch et al., 2016) e as figuras das estruturas moleculares otimizadas são geradas pelo software Avogadro software (Hanwell et al., 2012), devido à grande confiabilidade dos cálculos e qualidade gráfica das imagens. O programa Avogadro é um programa gratuito e com isso ele 
pode ser usado por alunos de universidades ou pesquisadores, sendo ele de fácil usabilidade. A Figura 5 mostra a simulação da adsorção de $\mathrm{CO}_{2}$ em uma amostra de hydrochar. Estudos de diferentes aspectos da catálise heterogênea apresentaram resultados importantes, usando-se dados da química quântica; como é mostrado no Quadro 4.

Figura 5 - Simulação computacional da adsorção de $\mathrm{CO}_{2}$ em uma amostra carbonácea com inserção de nitrogênio e oxigênio na molécula. Os átomos estão numerados e idetificados. Imagem gerada pelo programa Avogadro.

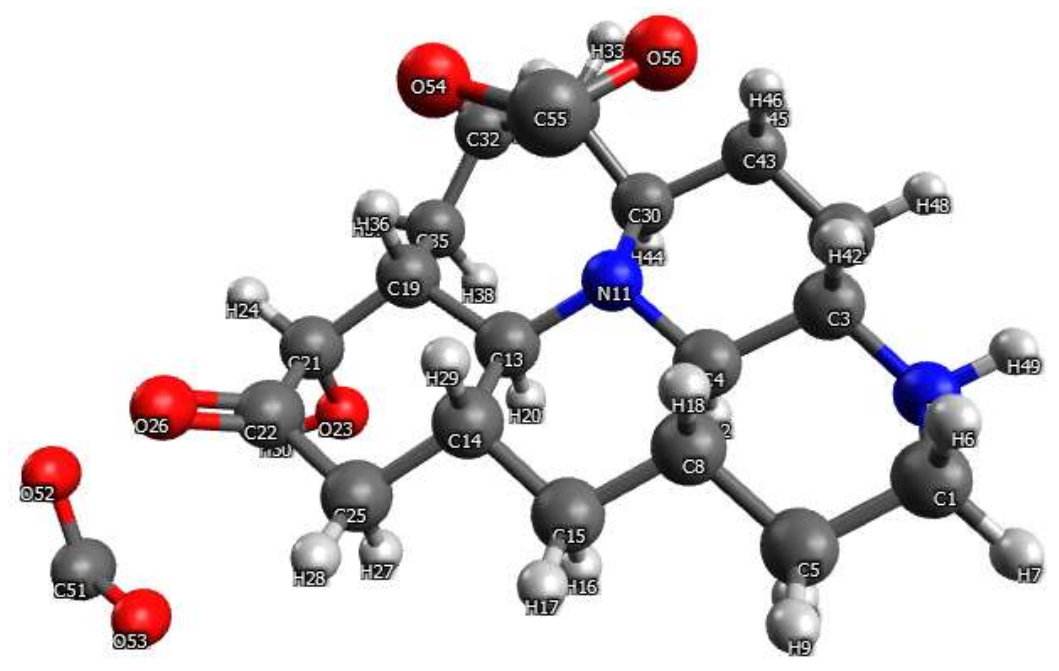

Fonte: Autores.

Quadro 4 - Estudos sobre atividade catalítica heterogênea e cálculos quânticos.

\begin{tabular}{|c|c|}
\hline Assunto & Autores \\
\hline $\begin{array}{l}\text { Adsorção de pequenas moléculas gasosas }\left(\mathrm{NH}_{3}, \mathrm{NO}, \mathrm{NO}_{2}, \mathrm{CO} \text { e } \mathrm{CO}_{2}\right) \text { em fosforeno negro, que } \\
\text { indicou que os cálculos cálculos em nível TFD mostram que todas as moléculas são adsorvidas } \\
\text { na superfície do fosforeno com diferentes sítios de adsorção favoráveis, dependendo da } \\
\text { geometria e orientação da molécula. }\end{array}$ & Sibari et al., 2021 \\
\hline $\begin{array}{l}\text { Adsorção de } \mathrm{H}_{2} \text { e } \mathrm{CO} \text { em ligas bimetálicas Ni-Au, sendo que os resultados do cálculo DFT } \\
\text { mostraram que a energia de adsorção de } \mathrm{CO} \text { nos locais superiores de Ni continua a diminuir com } \\
\text { o aumento da cobertura de Au devido ao efeito de conjunto geométrico conjugado. }\end{array}$ & Zhou et al., 2021 \\
\hline $\begin{array}{l}\text { Hidratação superficial de caolinita usando-se a TFD, que indicou que o mecanismo de hidratação } \\
\text { da superfície é principalmente que as moléculas de água na interface da caolinita são adsorvidas } \\
\text { na superfície com ligações de hidrogênio, e um filme hidratado composto de múltiplas moléculas } \\
\text { de água é gradualmente formado na superfície da caulinita com o aumento da taxa de cobertura } \\
\text { de água. }\end{array}$ & Chen et al., 2019 \\
\hline $\begin{array}{l}\text { Quantificar a área de superfície específica, distribuição de tamanho e volumes de poros por TFD } \\
\text { mostrou-se mais confiável a teoria experimental de Barrett-Joyner-Halenda. }\end{array}$ & Bardestani et al., 2019 \\
\hline $\begin{array}{l}\text { TFD foi usada para estudar os efeitos da rugosidade da superfície da membrana na incrustação } \\
\text { causada pela adesão de alginato . }\end{array}$ & R. Li et al., 2019 \\
\hline $\begin{array}{l}\text { A oxidação catalítica seletiva de } \mathrm{NH}_{3} \text { na presença de } \mathrm{SO}_{2} \text { por perovskitas modificadas contendo } \\
\text { lantânio e manganês foram estudadas por } \mathrm{TFD} \text {, que indicou que } \mathrm{NH}_{3} \text { é fortemente ligado aos } \\
\text { cátions das perovskitas. }\end{array}$ & Wang et al., 2018 \\
\hline $\begin{array}{l}\text { Cálculos em nível TFD para investigar o desempenho catalítico de um filme fino de } \mathrm{ZnO} \text { para a } \\
\text { oxidação de } \mathrm{NO} \text { e } \mathrm{CO} \text { para gerar } \mathrm{NO}_{2} \text { e } \mathrm{CO}_{2} \text { indicaram que a superfície sem defeitos de } \mathrm{ZnO} \\
\text { apresenta maior afinidade para o } \mathrm{NO} \text { nos três locais testados. No caso do oxigênio pré-adsorvido, } \\
\text { a superfície apresentou maiores energias de reação para o CO. }\end{array}$ & Galán \& Carbajal-Franco, 2021 \\
\hline
\end{tabular}

Fonte: Scholar Google. 
Os estudos sobre atividade catalítica indicaram sítios de maior reatividade, bem como as energias de adsorção, que são importantes para a compreensão e modelagem de futuros catalisadores, o que tem grande valor comercial, visto que a catálise heterogênea é um assunto de interesse por todo o mundo.

\subsection{Proteínas}

Proteínas são essenciais à vida e elas desempenham vários papéis importantes nos sistemas biológicos e alimentares, sendo que alguns deles são como: biocatalisadores, componentes estruturais de células e órgãos, proteínas contráteis, hormônios, proteínas de transporte quelantes de metal, anticorpos, proteínas de proteção e proteínas de armazenamento como nitrogênio e fonte de energia para embriões (Damodaran, 2017). O uso de TFD para estudos de proteínas tem grande importância, visto que, por se tratarem de moléculas com grande massa molecular e diferentes conformações, o estudo delas é bem interessante, pois cálculos de mecânica quântica podem ser aplicados para caracterização da configuração eletrônica dos fármacos estudados nos níveis atômico e molecular e as interações dos mesmos com proteínas (Khattab \& Al-Karmalawy, 2021). Além disso, cálculos realizados em nível TFD e uma avaliação completa do banco de dados estrutural de Cambridge e do banco de dados de proteínas foram conduzidos para avaliar a ocorrência e significância de interações intermoleculares com vários compostos nitro relevantes para a medicina (Hoffmann et al., 2020).

Em outro estudo, Neelakantan et al. (2018) sintetizaram complexos de $\mathrm{Cu}$ (II), $\mathrm{Ni}$ (II) e $\mathrm{Zn}$ (II) de (E) -2 - ((2,4dihidroxibenzilideno) amino) -3- (1H-indol-3-il) base de Schiff de ácido propanóico (L) e as geometrias moleculares dos complexos foram previstas otimizando a estrutura pelo método TFD/B3LYP com base LANL2DZ, definida na fase gasosa para estudar a interação dos complexos metálicos com ácido desoxirribonucléico de timo de vitelo e albumina de soro bovino.

Os cálculos quânticos confirmaram que as geometrias quadradas piramidais e trigonais bipiramidais caracterizadas cristalograficamente nos complexos 1 e 2 , respectivamente.

Devido a sua grande estrutura, proteínas dependem de uma variedade de interações não covalentes, sendo que a detecção direta e a caracterização dessas interações fracas são experimentalmente desafiadoras. Perras et al. (2017) relataram a primeira observação e medição de acoplamentos escalares "através do espaço" de longo alcance entre os grupos metil e carbonil da estrutura principal em proteínas. Esses acoplamentos J são indicativos da presença de interações não covalentes do tipo ligação de hidrogênio $\mathrm{C}-\mathrm{H} \cdots \pi$ envolvendo a rede amida $\pi$. Por meio da TFD, os acoplamentos escalares detectados experimentalmente foram corroborados pela análise orbital de ligação natural, que revelou a natureza orbital da interação e as origens dos acoplamentos " $\mathrm{J}$ " através do espaço. Esse estudo indicou que este tipo de interação $\mathrm{CH} \cdots \pi$ adiciona uma nova dimensão ao estudo da estrutura, função e dinâmica da proteína por espectroscopia de RMN.

Mesmo tratando-se de macromoléculas, vários aspectos das proteínas podem ser estudados usando-se cálculos quânticos, o que mostra a grande versatilidade do uso da TFD.

\section{Considerações Finais}

A química computacional tornou-se uma aliada importante para estudos em diversas áreas e os dados apresentados mostraram grande confiabilidade, comparados a dados experimentais. O seu uso em química analítica, em estudos de caráter ambiental e de superfícies catalíticas indicam que os fenômenos fisicoquímicos podem ser melhor compreendidos, visto que os sites reativos nas moléculas podem ter as reatividades simuladas computacionalmente. Mais que isso, ela pode ser usada para calcular os espectros vibracionais e os modos vibracionais normais para moléculas relativamente simples e o espectro infravermelho e Raman podem ser calculados e os resultados podem ser comparados com os dados experimentais e com isso a análise qualitativa de compostos desconhecidos se torna viável. Portanto, a química computacional iniciou como parte do estudo teórico do comportamento eletrônico e atualmente ela pode ser empregada para estudar particularidades em reações e 
comportamentos químicos, elucidação de estrutura molecular e muitos outros aspectos relacionados aos estudos moleculares. Portanto, a química computacional é uma importante ferramenta para a compreensão da complexidade mecanística devido à capacidade dela em obter informações sobre intermediários e estados de transição que são difíceis de caracterizar experimentalmente ou por meio de equipamentos. Espera-se que os cálculos quânticos sejam uma ferramenta de uso futuro cotidiano em diversos estudos, visto que pode-se reduzir o número de experimentos a serem executados, bem como melhorar a compreensão de vários comportamentos químicos, além de ajudar a planejar estudos de síntese orgânica e inorgânica.

\section{Agradecimentos}

À Faculdade de Farmácia e Instituto de Química da Universidade Federal de Goiás pelo apoio técnico e laboratorial. À Faculdade de Tecnologia, Engenharia Química e Ambiental da Universidade de Oulu-Finlândia. Aos coordenadores do programa "No Waste" no Brasil e Finlândia pela possibilidade de Intercâmbio na Universidade de Oulu e Coordenação de Aperfeiçoamento de Pessoal de Nível Superior (CAPES) para a concessão do doutorado.

\section{Referências}

Al-Hossainy, A. F., Abdelaal, R. M., \& El Sayed, W. N. (2021). Novel synthesis, structure characterization, DFT and investigation of the optical properties of cyanine dye/zinc oxide [4-CHMQI/ZnO] C nanocomposite thin film. Journal of Molecular Structure, 1224, 128989.

Alex, A., Harvey, S., Parsons, T., Pullen, F. S., Wright, P., \& Riley, J.-A. (2009). Can density functional theory (DFT) be used as an aid to a deeper understanding of tandem mass spectrometric fragmentation pathways? Rapid Communications in Mass Spectrometry, 23(17), 2619-2627. https://doi.org/10.1002/rcm.4163

Almlöf, J., \& Taylor, P. R. (1987). General contraction of Gaussian basis sets. I. Atomic natural orbitals for first-and second-row atoms. The Journal of Chemical Physics, 86(7), 4070-4077.

Almlöf, J., \& Taylor, P. R. (1991). Atomic natural orbital (ANO) basis sets for quantum chemical calculations. Advances in Quantum Chemistry, 22, $301-373$.

Almutairi, M. S., Zakaria, A. S., Ignasius, P. P., Al-Wabli, R. I., Joe, I. H., \& Attia, M. I. (2018). Synthesis, spectroscopic investigations, DFT studies, molecular docking and antimicrobial potential of certain new indole-isatin molecular hybrids: Experimental and theoretical approaches. Journal of Molecular Structure, 1153, 333-345. https://doi.org/https://doi.org/10.1016/j.molstruc.2017.10.025

Alsheikh, A. A., Žídek, J., Krčma, F., Papp, P., Lacko, M., \& Matejčík, Š. (2015). Fragmentation of methylphenylsilane and trimethylphenylsilane: A combined theoretical and experimental study. International Journal of Mass Spectrometry, 385, 1-12. https://doi.org/10.1016/j.ijms.2015.05.004

Antero, R. V. P., Alves, A. C. F., Ferreira Sales, P. D. T., de Oliveira, S. B., Ojala, S. A., \& Brum, S. S. (2019). A new approach to obtain mesoporousactivated carbon via hydrothermal carbonization of Brazilian Cerrado biomass combined with physical activation for bisphenol-A removal. Chemical Engineering Communications, 206(11). https://doi.org/10.1080/00986445.2019.1601625

Bahrami, H., Farajmand, B., \& Lakmehsari, M. S. (2018). Investigation of protonation and decomposition of tyrosine by ion mobility spectrometry and DFT calculations. International Journal of Mass Spectrometry, 430, 110-116.

Bakowies, D. (2007a). Accurate extrapolation of electron correlation energies from small basis sets. The Journal of Chemical Physics, $127(16)$, 164109.

Bakowies, D. (2007b). Extrapolation of electron correlation energies to finite and complete basis set targets. The Journal of Chemical Physics, $127(8), 84105$.

Bardestani, R., Patience, G. S., \& Kaliaguine, S. (2019). Experimental methods in chemical engineering: specific surface area and pore size distribution measurements-BET, BJH, and DFT. The Canadian Journal of Chemical Engineering, 97(11), 2781-2791. https://doi.org/https://doi.org/10.1002/cjce.23632

Barnes, L., Allouche, A.-R., Chambert, S., Schindler, B., \& Compagnon, I. (2020). Ion spectroscopy of heterogeneous mixtures: IRMPD and DFT analysis of anomers and conformers of monosaccharides. International Journal of Mass Spectrometry, 447, 116235.

Bartlett, R. J. (1995). Modern electronic structure theory. Yarkony, DR, Ed, 2, 10471131.

Başakçılardan Kabakcı, S., \& Baran, S. S. (2019). Hydrothermal carbonization of various lignocellulosics: Fuel characteristics of hydrochars and surface characteristics of activated hydrochars. Waste Management, 100, 259-268. https://doi.org/https://doi.org/10.1016/j.wasman.2019.09.021

Beć, K. B., Grabska, J., \& Czarnecki, M. A. (2018). Spectra-structure correlations in NIR region: spectroscopic and anharmonic DFT study of n-hexanol, cyclohexanol and phenol. Spectrochimica Acta Part A: Molecular and Biomolecular Spectroscopy, 197, $176-184$.

Beć, K. B., \& Huck, C. W. (2019). Breakthrough Potential in Near-Infrared Spectroscopy: Spectra Simulation. A Review of Recent Developments . In Frontiers in Chemistry (Vol. 7, p. 48). https://www.frontiersin.org/article/10.3389/fchem.2019.00048

Ben Said, R., Hamed, A. I., Mahalel, U. A., Al-Ayed, A. S., Kowalczyk, M., Moldoch, J., Oleszek, W., \& Stochmal, A. (2017). Tentative characterization of polyphenolic compounds in the male flowers of Phoenix dactylifera by liquid chromatography coupled with mass spectrometry and DFT. International Journal of Molecular Sciences, 18(3), 512. 
Brown, A. B., McKeogh, B. J., Tompsett, G. A., Lewis, R., Deskins, N. A., \& Timko, M. T. (2017). Structural analysis of hydrothermal char and its models by density functional theory simulation of vibrational spectroscopy. Carbon, 125, 614-629. https://doi.org/https://doi.org/10.1016/j.carbon.2017.09.051

Chen, J., Min, F., Liu, L., \& Liu, C. (2019). Mechanism research on surface hydration of kaolinite, insights from DFT and MD simulations. Applied Surface Science, 476, 6-15.

Chen, J., Min, F., Liu, L., Liu, C., \& Lu, F. (2017). Experimental investigation and DFT calculation of different amine/ammonium salts adsorption on kaolinite. Applied Surface Science, 419, 241-251.

Coolidge, A. S., \& James, H. M. (1938). Wave functions and potential curves for excited h2. The Journal of Chemical Physics, 6(11), 730-734.

Damodaran, S. (2017). Food proteins: an overview. Food Proteins and Their Applications, 1-24.

Daniel Boese, A., Klopper, W., \& Martin*, J. M. L. (2005). Anharmonic force fields and thermodynamic functions using density functional theory. Molecular Physics, 103(6-8), 863-876.

Ding, L., Wang, Z., Li, Y., Du, Y., Liu, H., \& Guo, Y. (2012). A novel hydrochar and nickel composite for the electrochemical supercapacitor electrode material. Materials Letters, 74, 111-114. https://doi.org/https://doi.org/10.1016/j.matlet.2012.01.070

Ding, L., Zou, B., Li, Y., Liu, H., Wang, Z., Zhao, C., Su, Y., \& Guo, Y. (2013). The production of hydrochar-based hierarchical porous carbons for use as electrochemical supercapacitor electrode materials. Colloids and Surfaces A: Physicochemical and Engineering Aspects, 423, 104-111. https://doi.org/https://doi.org/10.1016/j.colsurfa.2013.02.003

Du, Z., Huang, C., Meng, J., Yuan, Y., Yin, Z., Feng, L., Liu, Y., \& Zhang, L. (2020). Sorption of aromatic organophosphate flame retardants on thermally and hydrothermally produced biochars. Frontiers of Environmental Science \& Engineering, 14(3), 43. https://doi.org/10.1007/s11783-020-1220-6

Dunning Jr, T. H. (1989). Gaussian basis sets for use in correlated molecular calculations. I. The atoms boron through neon and hydrogen. The Journal of Chemical Physics, 90(2), 1007-1023.

Fan, Y., Liu, P.-F., Huang, Z.-Y., Jiang, T.-W., Yao, K.-L., \& Han, R. (2015). Porous hollow carbon spheres for electrode material of supercapacitors and support material of dendritic Pt electrocatalyst. Journal of Power Sources, 280, 30-38. https://doi.org/https://doi.org/10.1016/j.jpowsour.2015.01.096

Ferrari, B. C., \& Bennett, C. J. (2019). A Comparison of Medium-Sized Basis Sets for the Prediction of Geometries, Vibrational Frequencies, Infrared Intensities and Raman Activities for Water. Journal of Physics: Conference Series, 1290(1). https://doi.org/10.1088/1742-6596/1290/1/012013

Fonseca Guerra, C., Snijders, J. G., Te Velde, G., \& Baerends, E. J. (1998). Towards an order-N DFT method. Theoretical Chemistry Accounts, 99(6), 391403. https://doi.org/10.1007/s002140050353

Frisch, M. J., Trucks, G. W., Schlegel, H. B., Scuseria, G. E., Robb, M. A., Cheeseman, J. R., Scalmani, G., Barone, V., Petersson, G. A., \& Nakatsuji, H. (2016). Gaussian 16, Revision A. 03, Wallingford CT.

Futami, Y., Ozaki, Y., Hamada, Y., Wojcik, M. J., \& Ozaki, Y. (2011). Solvent dependence of absorption intensities and wavenumbers of the fundamental and first overtone of NH stretching vibration of pyrrole studied by near-infrared/infrared spectroscopy and DFT calculations. The Journal of Physical Chemistry A, 115(7), 1194-1198.

Galán, O. A. L., \& Carbajal-Franco, G. (2021). Energy profiles by DFT methods for CO and NO catalytic adsorption over ZnO surfaces. Catalysis Today, 360, 38-45. https://doi.org/https://doi.org/10.1016/j.cattod.2019.08.003

Gao, F., Shao, G., Qu, J., Lv, S., Li, Y., \& Wu, M. (2015). Tailoring of porous and nitrogen-rich carbons derived from hydrochar for high-performance supercapacitor electrodes. Electrochimica Acta, 155, 201-208. https://doi.org/https://doi.org/10.1016/j.electacta.2014.12.069

Geerlings, P., De Proft, F., \& Langenaeker, W. (2003). Conceptual Density Functional Theory. Chemical Reviews, 103(5), 1793-1874. https://doi.org/10.1021/cr990029p

Gómez, E. del V, Amaya-Roncancio, S., Avalle, L. B., Linares, D. H., \& Gimenez, M. C. (2017). DFT study of adsorption and diffusion of atomic hydrogen on metal surfaces. Applied Surface Science, 420, 1-8.

Grabska, J., Beć, K. B., Ishigaki, M., Huck, C. W., \& Ozaki, Y. (2018). NIR Spectra Simulations by Anharmonic DFT-Saturated and Unsaturated Long-Chain Fatty Acids. Journal of Physical Chemistry B, 122(27), 6931-6944. https://doi.org/10.1021/acs.jpcb.8b04862

Grabska, J., Beć, K. B., Ozaki, Y., \& Huck, C. W. (2017). Temperature drift of conformational equilibria of butyl alcohols studied by near-infrared spectroscopy and fully anharmonic DFT. The Journal of Physical Chemistry A, 121(9), 1950-1961.

Guangzhi, Y., Jinyu, Y., Yuhua, Y., Zhihong, T., DengGuang, Y., \& Junhe, Y. (2017). Preparation and CO 2 adsorption properties of porous carbon from camphor leaves by hydrothermal carbonization and sequential potassium hydroxide activation. RSC Advances, 7(7), 4152-4160.

Hanwell, M. D., Curtis, D. E., Lonie, D. C., Vandermeersch, T., Zurek, E., \& Hutchison, G. R. (2012). Avogadro: an advanced semantic chemical editor, visualization, and analysis platform. Journal of Cheminformatics, 4(1), 17. https://doi.org/10.1186/1758-2946-4-17

He, C., Giannis, A., \& Wang, J.-Y. (2013). Conversion of sewage sludge to clean solid fuel using hydrothermal carbonization: Hydrochar fuel characteristics and combustion behavior. Applied Energy, 111, 257-266. https://doi.org/https://doi.org/10.1016/j.apenergy.2013.04.084

Heilmann, S. M., Jader, L. R., Sadowsky, M. J., Schendel, F. J., von Keitz, M. G., \& Valentas, K. J. (2011). Hydrothermal carbonization of distiller's grains. Biomass and Bioenergy, 35(7), 2526-2533. https://doi.org/https://doi.org/10.1016/j.biombioe.2011.02.022

Helgaker, T., Jorgensen, P., \& Olsen, J. (2014). Molecular electronic-structure theory. John Wiley \& Sons. 
Helgaker, T., Klopper, W., \& Tew, D. P. (2008). Quantitative quantum chemistry. Molecular Physics, 106(16-18), 2107-2143. https://doi.org/10.1080/00268970802258591

Hoffmann, J. M., Sadhoe, A. K., \& Mooibroek, T. J. (2020). $\pi$-Hole Interactions with Various Nitro Compounds Relevant for Medicine: DFT Calculations and Surveys of the Cambridge Structural Database (CSD) and the Protein Data Bank (PDB). Synthesis, 52(04), 521-528.

Huck, C. W. (2017). Selected latest applications of molecular spectroscopy in natural product analysis. Phytochemistry Letters, 20, 491-498.

Ibrahim, S. M., \& Al-Hossainy, A. F. (2021). Synthesis, structural characterization, DFT, kinetics and mechanism of oxidation of bromothymol blue: application to textile industrial wastewater treatment. Chemical Papers, 75(1), 297-309. https://doi.org/10.1007/s11696-020-01299-8

Imamura, Y., \& Scuseria, G. E. (2003). A new correlation functional based on a transcorrelated Hamiltonian. The Journal of Chemical Physics, 118(6), 24642469.

Jain, A., Balasubramanian, R., \& Srinivasan, M. P. (2016). Hydrothermal conversion of biomass waste to activated carbon with high porosity: A review. Chemical Engineering Journal, 283, 789-805. https://doi.org/https://doi.org/10.1016/j.cej.2015.08.014

Jamaludin, N., Tan, T. L., Zaman, A. S. K., Sadrolhosseini, A. R., \& Rashid, S. A. (2020). Empty Fruit Bunch Biochar. Materials, 13, 33556.

James, H. M., \& Coolidge, A. S. (1936). On the ground state of lithium. Physical Review, 49(9), 688.

Ji, Y., Yang, X., Ji, Z., Zhu, L., Ma, N., Chen, D., Jia, X., Tang, J., \& Cao, Y. (2020). DFT-Calculated IR Spectrum Amide I, II, and III Band Contributions of N-Methylacetamide Fine Components. ACS Omega, 5(15), 8572-8578. https://doi.org/10.1021/acsomega.9b04421

Jinnouchi, R., \& Asahi, R. (2017). Predicting Catalytic Activity of Nanoparticles by a DFT-Aided Machine-Learning Algorithm. The Journal of Physical Chemistry Letters, 8(17), 4279-4283. https://doi.org/10.1021/acs.jpclett.7b02010

Kenny, P. T. M., Nomoto, K., \& Orlando, R. (1992). Fragmentation studies of peptides: The formation of Y ions. Rapid Communications in Mass Spectrometry, 6(2), 95-97. https://doi.org/10.1002/rcm.1290060205

Khattab, M., \& Al-Karmalawy, A. A. (2021). Revisiting Activity of Some Nocodazole Analogues as a Potential Anticancer Drugs Using Molecular Docking and DFT Calculations. Frontiers in Chemistry, 9(March), 1-10. https://doi.org/10.3389/fchem.2021.628398

Köche, J. C. (2016). Fundamentos de metodologia científica. Editora Vozes.

Kutzelnigg, W., \& Morgan III, J. D. (1992). Erratum: Rates of convergence of the partial-wave expansions of atomic correlation energies [J. Chem. Phys. 96, 4484 (1992)]. The Journal of Chemical Physics, 97(11), 8821.

Lee, J., Kim, Y., Kim, W. Y., \& Oh, H. Bin. (2020). Graph theory-based reaction pathway searches and DFT calculations for the mechanism studies of free radical-initiated peptide sequencing mass spectrometry (FRIPS MS): a model gas-phase reaction of GGR tri-peptide. Physical Chemistry Chemical Physics, 22(9), 5057-5069.

Lee, S.-S., Lee, J., Oh, J. H., Park, S., Hong, Y., Min, B. K., Lee, H. H. L., Kim, H. I., Kong, X., \& Lee, S. (2018). Chiral differentiation of D-and L-isoleucine using permethylated $\beta$-cyclodextrin: Infrared multiple photon dissociation spectroscopy, ion-mobility mass spectrometry, and DFT calculations. Physical Chemistry Chemical Physics, 20(48), 30428-30436.

Li, R., Lou, Y., Xu, Y., Ma, G., Liao, B.-Q., Shen, L., \& Lin, H. (2019). Effects of surface morphology on alginate adhesion: Molecular insights into membrane fouling based on XDLVO and DFT analysis. Chemosphere, 233, 373-380. https://doi.org/https://doi.org/10.1016/j.chemosphere.2019.05.262

Li, X., Zhang, R., Zhu, X., \& Zhang, L. (2020). Effect of N-doping on the catalytic decomposition of hydrogen iodide over activated carbon: Experimental and DFT studies. International Journal of Hydrogen Energy, 45(7), 4511-4520.

Lin, Y., Ma, X., Peng, X., Hu, S., Yu, Z., \& Fang, S. (2015). Effect of hydrothermal carbonization temperature on combustion behavior of hydrochar fuel from paper sludge. Applied Thermal Engineering, 91, 574-582. https://doi.org/https://doi.org/10.1016/j.applthermaleng.2015.08.064

Löwdin, P.-O. (1955). Quantum theory of many-particle systems. I. Physical interpretations by means of density matrices, natural spin-orbitals, and convergence problems in the method of configurational interaction. Physical Review, 97(6), 1474.

Maclot, S., Piekarski, D. G., Domaracka, A., Méry, A., Vizcaino, V., Adoui, L., Martín, F., Alcamí, M., Huber, B. A., Rousseau, P., \& Díaz-Tendero, S. (2013). Dynamics of Glycine Dications in the Gas Phase: Ultrafast Intramolecular Hydrogen Migration versus Coulomb Repulsion. The Journal of Physical Chemistry Letters, 4(22), 3903-3909. https://doi.org/10.1021/jz4020234

Mäkelä, M., Benavente, V., \& Fullana, A. (2015). Hydrothermal carbonization of lignocellulosic biomass: Effect of process conditions on hydrochar properties. Applied Energy, 155, 576-584. https://doi.org/https://doi.org/10.1016/j.apenergy.2015.06.022

Modesto-Costa, L., Martinez, S. T., Pinto, A. C., Vessecchi, R., \& Borges Jr, I. (2018). Elucidating the mass spectrum of the retronecine alkaloid using DFT calculations. Journal of Mass Spectrometry, 53(10), 934-941.

Morris, R. E., \& Wheatley, P. S. (2008). Gas Storage in Nanoporous Materials. Angewandte Chemie International Edition, 47(27), 4966-4981. https://doi.org/10.1002/anie.200703934

Naseem, S., Khalid, M., Tahir, M. N., Halim, M. A., Braga, A. A. C., Naseer, M. M., \& Shafiq, Z. (2017). Synthesis, structural, DFT studies, docking and antibacterial activity of a xanthene based hydrazone ligand. Journal of Molecular Structure, 1143(November), 235-244. https://doi.org/10.1016/j.molstruc.2017.04.093 
Neelakantan, M. A., Balamurugan, K., Balakrishnan, C., \& Subha, L. (2018). Interaction of amino acid Schiff base metal complexes with DNA/BSA protein and antibacterial activity: spectral studies, DFT calculations and molecular docking simulations. Applied Organometallic Chemistry, 32 (4), e4259.

Ozaki, Y., \& Kawata, S. (1996). Near-infrared spectroscopy. Gakkai Shuppan Center, Tokyo, Japan.

Pereira, A. S., Shitsuka, D. M., Parreira, F. J., \& Shitsuka, R. (2018). Metodologia da pesquisa científica. UFSM. Disponível em: https://repositorio. ufsm. $\mathrm{br} / \mathrm{bitstream} / \mathrm{handle}$

Perras, F. A., Marion, D., Boisbouvier, J., Bryce, D. L., \& Plevin, M. J. (2017). Observation of CH $\cdots \pi$ Interactions between Methyl and Carbonyl Groups in Proteins. Angewandte Chemie, 129(26), 7672-7675.

Polyansky, O. L., Császár, A. G., Shirin, S. V, Zobov, N. F., Barletta, P., Tennyson, J., Schwenke, D. W., \& Knowles, P. J. (2003). High-accuracy ab initio rotation-vibration transitions for water. Science, 299(5606), 539-542.

Pople, J. A., Head-Gordon, M., \& Raghavachari, K. (1987). Quadratic configuration interaction. A general technique for determining electron correlation energies. The Journal of Chemical Physics, 87(10), 5968-5975.

Prashantha, A. G., Ali, R. A. S., \& Keshavayya, J. (2021). Synthesis, spectral characterization, DFT studies and antimicrobial activities of aminomethylbenzoic acid based azo dyes. Inorganic Chemistry Communications, 127, 108392.

Rad, A. S., Ardjmand, M., Esfahani, M. R., \& Khodashenas, B. (2021). DFT calculations towards the geometry optimization, electronic structure, infrared spectroscopy and UV-vis analyses of Favipiravir adsorption on the first-row transition metals doped fullerenes; a new strategy for COVID-19 therapy. Spectrochimica Acta Part A: Molecular and Biomolecular Spectroscopy, 247, 119082.

Shahana, M. F., \& Yardily, A. (2020). Synthesis, spectral characterization, DFT, and docking studies of (4-amino-2-(phenylamino) thiazol-5-yl)(thiophene-2yl) methanone and (4-amino-2-(4-chlorophenyl) amino) thiazol-5-yl)(thiophene-2-yl) methanone. Journal of Structural Chemistry, 61(9), $1367-1379$.

Shao, P., Pei, J., Tang, H., Yu, S., Yang, L., Shi, H., Yu, K., Zhang, K., \& Luo, X. (2021). Defect-rich porous carbon with anti-interference capability for adsorption of bisphenol A via long-range hydrophobic interaction synergized with short-range dispersion force. Journal of Hazardous Materials, 403(July 2020), 123705. https://doi.org/10.1016/j.jhazmat.2020.123705

Sharma, Sanjeev, \& Chun, S. E. (2019). New high-yield method for the production of activated carbon via hydrothermal carbonization (HTC) processing of carbohydrates. Journal of Electrochemical Science and Technology, 10(4), 387-393. https://doi.org/10.33961/jecst.2019.00059

Sharma, Shikha, Dumpalan, R. M. R., \& Rawat, N. (2020). Experimental and DFT studies on complexation of uranyl with N-(2-Hydroxyethyl) iminodiacetic acid in aqueous medium. Inorganica Chimica Acta, 508, 119653.

Shi, L., Meng, S., Jungsuttiwong, S., Namuangruk, S., Lu, Z.-H., Li, L., Zhang, R., Feng, G., Qing, S., \& Gao, Z. (2020). High coverage H2O adsorption on CuAl2O4 surface: A DFT study. Applied Surface Science, 507, 145162.

Sibari, A., Kerrami, Z., Benaissa, M., \& Kara, A. (2021). Coverage-dependent adsorption of small gas molecules on black phosphorene: a DFT study. Surface Science, 710, 121860. https://doi.org/https://doi.org/10.1016/j.susc.2021.121860

Soleimani, F., Karimi, R., \& Gharib, F. (2016). Thermodynamic Studies on Protonation Constant of Acyclovir at Different Ionic Strengths. Journal of Solution Chemistry, 45(6), 920-931. https://doi.org/10.1007/s10953-016-0478-6

Song, X., Ning, P., Li, K., Sun, X., Wang, C., \& Sun, L. (2018). Hydrogen transfer effect and reaction mechanism for catalytic hydrolysis of HCN in ionic liquids: A density functional theory study. Chemical Engineering Journal, 348, 630-636. https://doi.org/https://doi.org/10.1016/j.cej.2018.05.056

Szalewicz, K., Jeziorski, B., Monkhorst, H. J., \& Zabolitzky, J. G. (1982). A new functional for variational calculation of atomic and molecular second-order correlation energies. Chemical Physics Letters, 91(3), 169-172.

Takahashi, H., \& Yabushita, S. (2013). Theoretical analysis of weak adjacent substituent effect on the overtone intensities of XH (X= C, O) stretching vibrations. The Journal of Physical Chemistry A, 117(26), 5491-5502.

Thomas, R., Hossain, M., Mary, Y. S., Resmi, K. S., Armaković, S., Armaković, S. J., Nanda, A. K., Ranjan, V. K., Vijayakumar, G., \& Van Alsenoy, C. (2018). Spectroscopic analysis and molecular docking of imidazole derivatives and investigation of its reactive properties by DFT and molecular dynamics simulations. Journal of Molecular Structure, 1158, 156-175. https://doi.org/https://doi.org/10.1016/j.molstruc.2018.01.021

Titirici, M.-M., Thomas, A., \& Antonietti, M. (2007). Back in the black: hydrothermal carbonization of plant material as an efficient chemical process to treat the CO2 problem? New Journal of Chemistry, 31(6), 787-789. https://doi.org/10.1039/B616045J

Vessecchi, R., da Silva Borges, L., da Silva Emery, F., \& Lopes, N. P. (2017). Understanding the fragmentation mechanisms of methoxy-, mesyl-, and tosyllapachol derivatives by computational chemistry and mass spectrometry analysis. International Journal of Mass Spectrometry, 418, 92-100. https://doi.org/10.1016/j.ijms.2016.11.012

Wang, D., Peng, Y., Yang, Q., Xiong, S., Li, J., \& Crittenden, J. (2018). Performance of Modified LaxSr1-xMnO3 Perovskite Catalysts for NH3 Oxidation: TPD, DFT, and Kinetic Studies. Environmental Science \& Technology, 52(13), 7443-7449. https://doi.org/10.1021/acs.est.8b01352

Wu, J., Yang, J., Huang, G., Xu, C., \& Lin, B. (2020). Hydrothermal carbonization synthesis of cassava slag biochar with excellent adsorption performance for Rhodamine B. Journal of Cleaner Production, 251, 119717. https://doi.org/https://doi.org/10.1016/j.jclepro.2019.119717

Xiao, L.-P., Shi, Z.-J., Xu, F., \& Sun, R.-C. (2012). Hydrothermal carbonization of lignocellulosic biomass. Bioresource Technology, 118, 619-623. https://doi.org/https://doi.org/10.1016/j.biortech.2012.05.060

Yang, S., Lei, G., Xu, H., Xu, B., Li, H., Lan, Z., Wang, Z., \& Gu, H. (2019). A DFT study of CO adsorption on the pristine, defective, In-doped and Sb-doped 
Research, Society and Development, v. 10, n. 8, e45910817567, 2021

(CC BY 4.0) | ISSN 2525-3409 | DOI: http://dx.doi.org/10.33448/rsd-v10i8.17567

graphene and the effect of applied electric field. Applied Surface Science, 480, 205-211.

Zhou, L., Yang, Y., Chen, J., Qiu, R., \& Yao, Y. (2021). CO and H2 adsorption on Au-Ni bimetallic surfaces: a combined experimental and DFT theoretical study. Surface Science, 121892. 